\title{
Fatty Acid Composition of Adipose Tissue Triglycerides in Obese Diabetic Women After Bariatric Surgery: a 2-Year Follow up
}

\author{
M. KUNEŠOVÁ ${ }^{1,2}$, B. SEDLÁČKOVÁ ${ }^{1}$, O. BRADNOVÁ ${ }^{1}$, E. TVRZICKÁ ${ }^{2}$, \\ B. STAŇKOVÁ ${ }^{2}$, P. ŠRÁMKOVÁ ${ }^{3}$, K. DOLEŽALOVÁ ${ }^{3,4}$, P. KALOUSKOVÁ $^{1}$, \\ P. HLAVATÝ ${ }^{1,3}$, M. HILL ${ }^{1}$, B. BENDLOVÁ ${ }^{1}$, M. FRIED ${ }^{3,4}$, V. HAINER ${ }^{1}$, J. VRBÍKOVÁ ${ }^{1}$ \\ ${ }^{1}$ Institute of Endocrinology Obesity Management Centre, Prague, Czech Republic, ${ }^{2}$ Fourth \\ Department of Internal Medicine, First Medical Faculty, Charles University, Prague, Czech \\ Republic, ${ }^{3}$ OB Clinic, Prague, Czech Republic, ${ }^{4}$ First Medical Faculty, Charles University, Prague, \\ Czech Republic
}

Received July 13, 2015

Accepted July 27, 2015

\begin{abstract}
Summary
Bariatric surgery is the most effective method in the treatment of obesity and type 2 diabetes (T2DM). The aim of this study was to evaluate the effects of different types of bariatric procedures on remission of T2DM and on the fatty acid composition in subcutaneous adipose tissue. Patients included obese diabetic women who underwent bariatric surgery: biliopancreatic diversion (BPD), $n=8$, laparoscopic gastric banding (LAGB), $n=9$ or laparoscopic greater curvature plication (LGCP), $n=12$. Anthropometric characteristics and fatty acid composition of adipose tissue (FA AT) were analyzed before surgery, then 6 months and 2 years after surgery. FA AT was analyzed by gas chromatography. Diabetes remission was estimated. BPD was most efficient in inducing a remission of diabetes $(p=0.004)$. Significantly higher increases in lauric (12:0), myristoleic (14:1n-5) and palmitoleic (16:1n-7) acids and delta-9 desaturase were found two years after BPD, suggesting higher lipogenesis in adipose tissue. Docosatetraenoic acid (22:4n-6) increased significantly after BPD, while docosapentaenoic acid (22:5n-3) decreased 6 months after BPD and increased after 2 years. No changes were found after LAGB and LGCP after 2 years. Bariatric surgery led to significant changes in the fatty acid composition of subcutaneous adipose tissue in severely obese diabetic women after six months and two years, and was partly influenced by the type of surgery used.
\end{abstract}

\section{Key words}

Obesity • Adipose tissue • Bariatric surgery • Palmitoleic acid • Fatty acids

\section{Corresponding author}

M. Kunešová, Institute of Endocrinology, Národní 8, 11694 Prague 1, Czech Republic. E-mail: mkunesova@endo.cz

\section{Introduction}

Bariatric surgery is the most efficient method for the management of type 2 diabetes mellitus in obese subjects (Buchwald et al. 2004, 2009, Scheen et al. 2009). Various methods are used for the bariatric management of obese diabetics, with the differences of individual surgical procedures resulting in different weight loss and metabolic changes. Biliopancreatic diversion (BPD) is the most effective metabolic surgical procedure due to its mechanism of action including effects on the incretin production of GLP 1 (Tsoli et al. 2013), bile acid secretion (Ferrannini et al. 2015), and gut microbiota (Calvani et al. 2010, Clemente-Postigo et al. 2015). Acute caloric restriction may also be involved (Plourde et al. 2014). Laparoscopic adjustable gastric banding (LAGB) is one of the most frequently used restrictive methods, and its effect is mediated mainly by weight loss (Svane and Madsbad 2014). Laparoscopic greater curvature plication (LGCP) was recently introduced as a method of metabolic surgery (Talebpour et al. 2012, Fried et al. 2012). Subsequent decreases in ghrelin and increases in GIP could make this method effective in treating type 2 diabetes (Bradnova et al. 2014).

Obesity and type 2 diabetes are often associated with dyslipidemia and an altered composition of fatty

PHYSIOLOGICAL RESEARCH • ISSN 0862-8408 (print) • ISSN 1802-9973 (online)

(c) 2015 Institute of Physiology v.v.i., Academy of Sciences of the Czech Republic, Prague, Czech Republic

Fax +420 241062 164, e-mail: physres@biomed.cas.cz, www.biomed.cas.cz/physiolres 
acids in serum and adipose tissue lipids. The fatty acid composition of adipose tissue triglycerides (AT TG) reflects not only the composition of fat in food, but also the metabolic processing of fat, such as endogenous lipogenesis and lipid oxidation.

The aim of this study was to compare effect of three bariatric methods - biliopancreatic diversion (BPD), laparoscopic adjustable gastric banding (LAGB) and laparoscopic greater curvature plication (LGCP) on serum lipids and the composition of adipose triglycerides in obese type 2 diabetic women after 6 months and 2 years. The effect on glucose metabolism will be presented elsewhere.

\section{Methods}

Subjects

The study included 29 morbidly obese women with T2DM (age: 39-66 years; duration of diabetes: 1-14 years) that underwent BPD (8 subjects), LAGB (9 subjects) or LGCP (12 subjects). The patients were enrolled over time, as they were indicated for the operations. T2DM patients eligible for bariatric surgery were allocated to different bariatric procedures according to the consecutive numbers they received as they entered the process for study enrolment. Antidiabetic treatment for the patients was as follows: diet only in 3 cases, metformin in 26 cases, sulphonylurea in 8 cases and insulin in 3 cases. Exclusion criteria were treatment with either glitazones, DPP-IV inhibitors or GLP1 agonists, active cancer or an acute medical condition requiring hospitalization, evidence or history of clinically significant cardiovascular, pulmonary, endocrine (other than obesity and T2DM), hematological, renal, gastrointestinal, hepatic (other than NAFLD), neurologic, psychiatric, or severe allergic disease, pregnancy, breastfeeding, weight change more than $5 \%$ of body weight over the preceding 12 weeks, or recent changes in exercise intensity or frequency over the preceding 4 weeks. All subjects signed an informed consent approved by the local ethical committee before entering the study.

\section{Study design}

One month before entering the study the subjects were put on a hypocaloric diet with $5000 \mathrm{~kJ} /$ day. Oral antidiabetic drugs were discontinued 3 days before examinations and long-acting insulin $24 \mathrm{~h}$ before examinations.
The following bariatric procedures were performed: laparoscopic adjustable gastric banding, laparoscopic greater curvature plication and biliopancreatic diversion.

\section{Body composition}

Anthropometric measurements were performed for all patients. Body weight was measured to the nearest $0.5 \mathrm{~kg}$ and height to the nearest $1 \mathrm{~cm}$. BMI was calculated as body weight in kilograms divided by the square of the height in meters. Waist circumference was measured in the standing position, at half of the distance between the lower ribs and the crest of the pelvis. Hip circumference was measured as the widest gluteal circumference. Body composition was assessed by DEXA (GE LUNAR iDXA, GE Healthcare Technology, USA).

\section{Adipose fatty acid analysis}

Total lipids were extracted from 3-5 mg of adipose tissue by the method of Folch and coworkers (Folch et al. 1957) using dichlormethane instead of chloroform (Carlson 1985). Samples were transmethylated to FAME with $1 \mathrm{M}$ sodium methoxide in dry methanol under nitrogen atmosphere in darkness, for $60 \mathrm{~min}$ at ambient temperature. The reaction mixture was then neutralized with $1 \mathrm{M}$ acetic acid, methyl esters were extracted into hexane and passed through a column $(5 \times 20 \mathrm{~mm})$ of anhydrous sodium sulphate. Extracts were dried under nitrogen, dissolved in an appropriate volume of isooctane and stored at $-80^{\circ} \mathrm{C}$ until analyzed.

Gas chromatography was performed with a Trace GC gas chromatograph combined with an AS 2000 autosampler (Thermo Finnigan, USA). The chromatograph was equipped with a capillary split/splitless injector and flame ionization detector (FID).

Analysis of FAME was performed on a fusedsilica capillary column coated with $0.25 \mu \mathrm{m}$ chemically bonded stationary phase Select FAME (100 m, $0.25 \mathrm{~mm}$ I.D., Agilent Technologies, The Netherlands). The oven temperature was programmed from $80^{\circ} \mathrm{C}$ to $120^{\circ} \mathrm{C}$ at $4 \% \mathrm{~min}$, to $270^{\circ} \mathrm{C}$ at $2 \% \mathrm{~min}$, then isothermal for $25 \mathrm{~min}$. The injector and detector temperatures were 250 and $270{ }^{\circ} \mathrm{C}$, respectively. Hydrogen carrier gas was maintained at a head pressure of $70 \mathrm{kPa}$ and split flow $10 \mathrm{ml} / \mathrm{min}$, splitless time $0.25 \mathrm{~min}$.

Integration software Clarity (Data Apex Ltd. Prague, Czech Republic) was used for data acquisition and handling. 


\section{Plasma samples}

Blood samples were collected into chilled EDTA-containing tubes for assessment of glucose, insulin C-peptide levels, hsCRP and blood lipids. All samples were immediately cooled, and plasma was prepared, aliquoted, and stored at $-80{ }^{\circ} \mathrm{C}$ until assayed. Plasma levels of blood glucose, insulin, C-peptide and HbA1c were measured using the Cobas 6000 analyzer. Plasma concentrations of serum lipids and hsCRP were analyzed using standard laboratory methods.

\section{Statistics}

The relationships between individual metric variables and factors were evaluated by ANOVA models followed by least significant difference multiple comparisons. The model consisted of a subject factor (separating inter-individual variability from the remaining factors), the between-subject factors Operation, Age over median, BMI over median, initial value of dependent variable over median, within-subject factor Exam and Operation $\times$ Exam interaction. The original data were transformed by power transformations to attain symmetric data distribution and constant variance (Meloun et al. 2000). The homogeneity of data was checked using residual analysis as explained elsewhere (Meloun et al. 2002, 2004). These analyses were performed in Statgraphics Centurion v. XV (Statpoint Inc., Herndon, Maryland, USA) statistical software.

The statistical software SIMCA-P+ Version 12.0.0.0 from Umetrics AB (Umeå, Sweden) was used for further data analysis. This software enabled us to find the number of the relevant components utilizing the prediction error sum of squares, and also allowed the detection of multivariate non-homogeneities and testing the multivariate normal distribution and homoscedasticity (homogeneity of variance).

The relationships between diabetes remission after individual bariatric procedures was tested using log-linear model (frequency analysis) using statistical software NCSS 2007, Kaysville, Utah, USA.

The results are given as means (lower limit of CI; upper limit of CI at the $95 \%$ level) unless stated otherwise.

Due to significant differences between groups the results were evaluated after adjustment for age, BMI and initial level of the variable.

Diabetes remission was estimated according to Buse et al. (2009).

The study was approved by the Ethical Committee of the Institute of Endocrinology in Prague.

\section{Results}

Changes of anthropometric parameters and body composition after individual procedures are shown in Table 1. Significant differences in weight and body fat loss among individual types of surgeries were found, with the highest loss observed after BPD as expected due to the mechanism of action of this method.
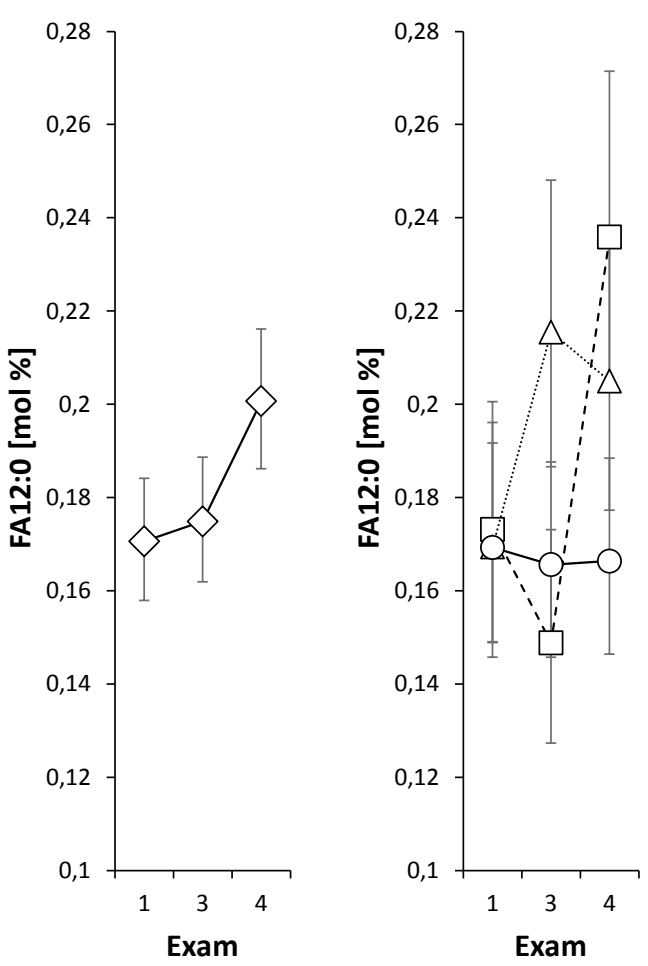

Fig. 1a. Lauric acid (12:0) in adipose tissue before the surgery (1), after 6 months (3) and after 2 years (4). Squares BPD, triangles LAGB, circles LGCP. Exam: $F=2.7, p=0.073$, Oper $x$ Exam: $F=2.7, p=0.037$.

Changes in the individual fatty acid composition of adipose tissue triglycerides, blood lipids and hsCRP are given in Tables 2-4. D-6 desaturase activity calculated as a product/precursor ratio did not differ significantly (data not shown). When evaluating differences between individual bariatric procedures we found significant differences in the following saturated and monounsaturated fatty acids: lauric acid (12:0), myristoleic acid (14:1n-5), palmitoleic acid (16:1n-7) and stearic acid (18:0) (Table 2, Fig. 1a and 1b). Concurrently, a significant difference was found in the activity of stearoyl-CoA desaturase-1 (SCD1, delta-9 desaturase) calculated as product/precursor ratio (14:1n-5/14:0, D9-14, p=0.014, 18:1n-9/18:0, D9-18, $\mathrm{p}=0.021$ ) (Fig. 2). After BPD, there was an increase in the 
percentage of lauric, myristoleic and palmitoleic acid after 2 years concurrent with an increase SCD1 activity, suggesting increased lipogenic activity 2 years after the surgery. After LAGB, increased levels were found 6 months after surgery followed mostly by a decrease to the initial levels after 2 years. Gastric plication did not induce significant changes.

For polyunsaturated fatty acids we found significant differences among individual bariatric methods in $n-6$ and n-3 polyunsaturated fatty acids.
Significant differences were found in changes of docosatetraenoic acid (22:4n-6, DTA) and in docosapentaenoic acid (22:5n-3, DPA) (Table 3, Fig. 3). In the BPD group, there was an increase in DTA two years after the surgery, while no change was found in subjects after LAGB and LGCP. Representative of n-3 PUFA, DPA decreased after 6 months in the BPD and LAGB groups, followed by an increase after 2 years. In LGCP group the level of DPA did not change (Table 3, Fig. 3).
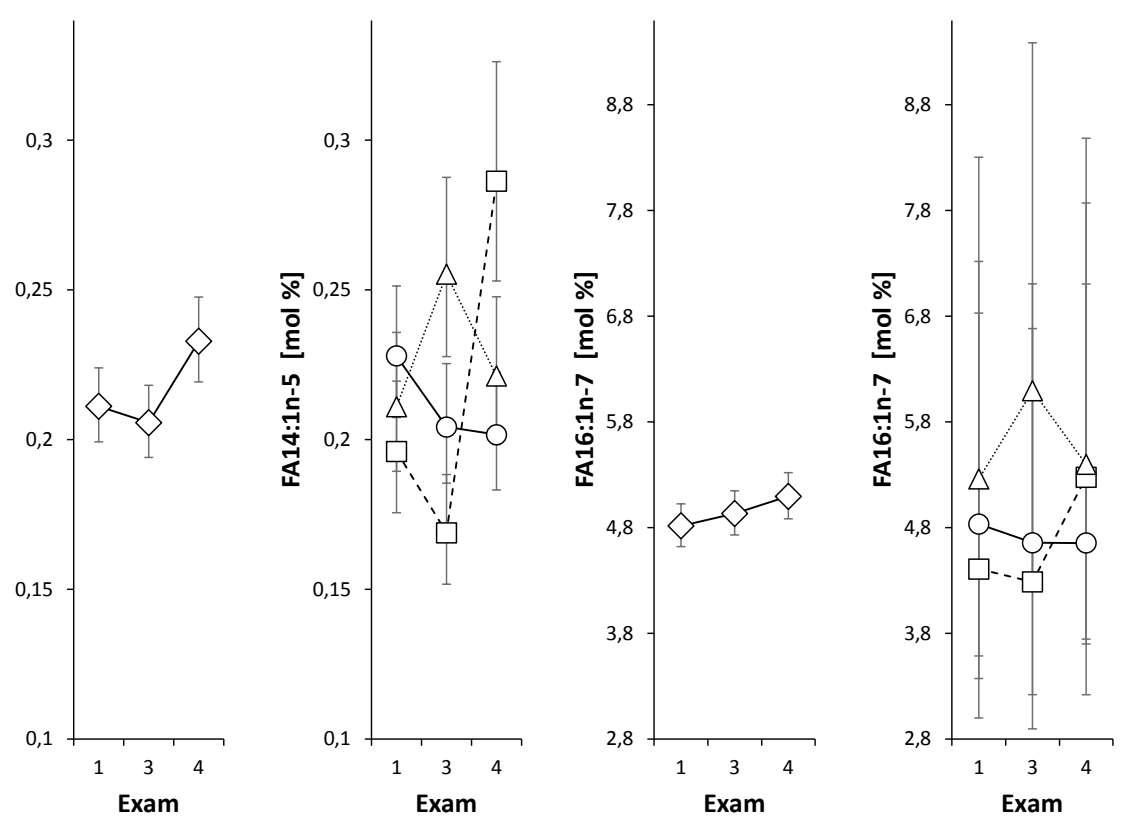

Fig. 1b. Myristoleic (14:1n-5) and palmitoleic (16:1n-7) acid in adipose tissue before the surgery (1), after 6 months (3) and after 2 years (4). Squares BPD, triangles LAGB, circles LGCP. 14:1n-5: Exam: $F=2.5, p=0.094$, Oper x Exam: $F=6.7, p<0.001$. 16:1n-7: Exam: $\mathrm{F}=0.9, \mathrm{p}=412$, Oper $x$ Exam: $\mathrm{F}=3.0, \mathrm{p}=0.027$.
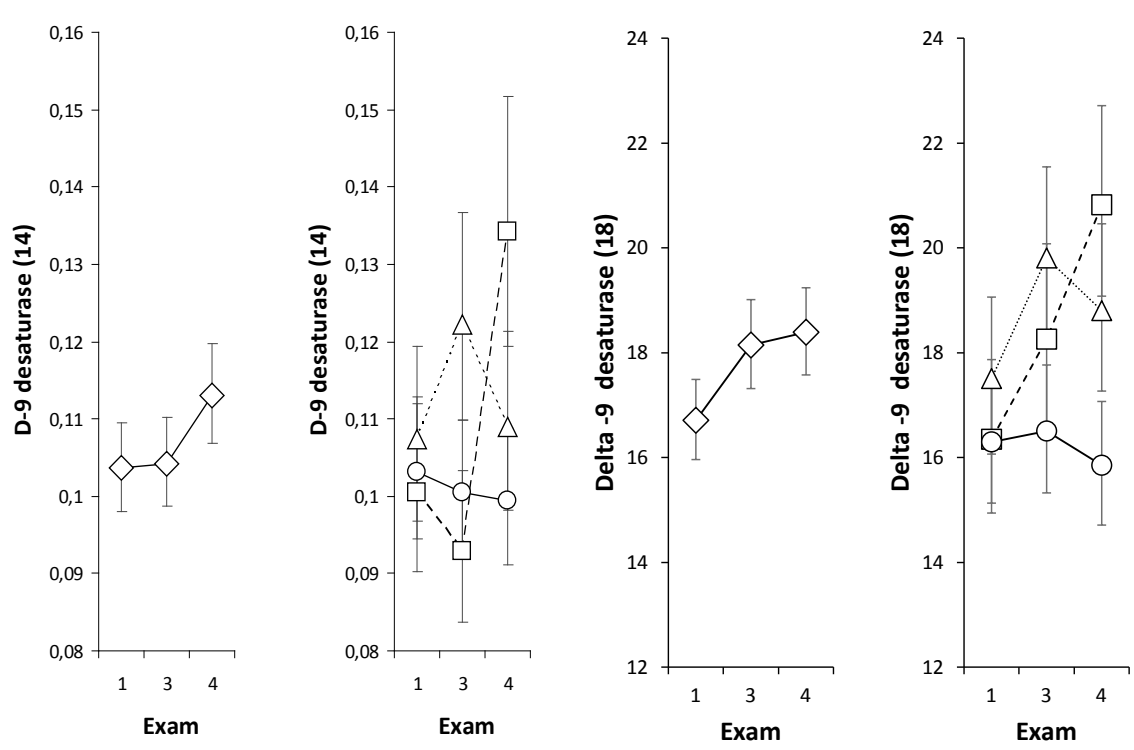

Fig. 2. Delta- 9 desaturase $14: 1 n-5 / 14: 0$ and $18: 1 n-9 / 18: 0$ in adipose tissue before the surgery (1), after 6 months (3) and after 2 years (4). Squares BPD, triangles LAGB, circles LGCP. Delta-9 desaturase (14:1n-5/14:0): Exam: $F=1.5, p=0.227$, Oper $x$ Exam: $F=3.4, p=0.014$. Delta-9 desaturase $(18: 1 n-9 / 18: 0)$ : Exam: $F=F=4.6, p=0.013$, Oper $x$ Exam: $F=3.1, p=0.021$. 

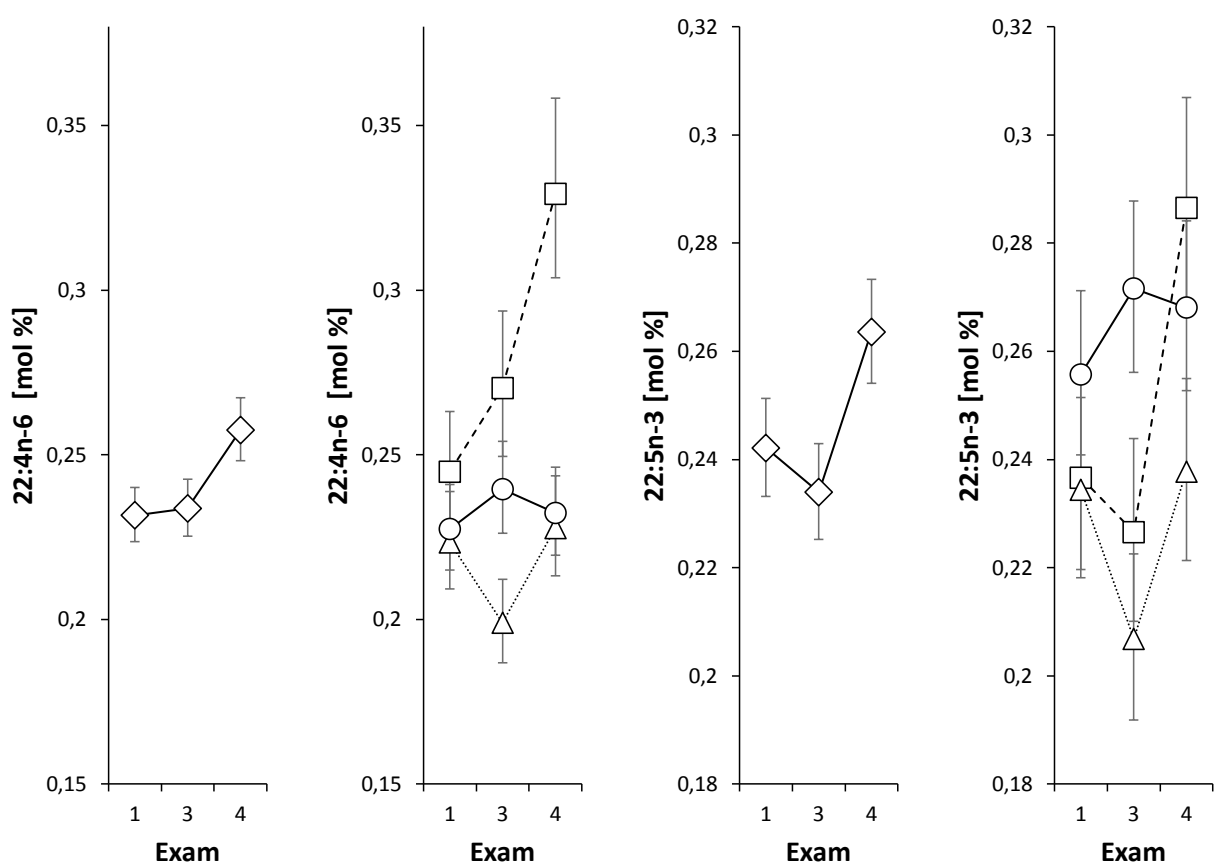

Fig. 3. Docosatetreaenoic (22:4n-6) and docosapentaenoic (22:5n-3) acid in adipose tissue before the surgery (1), after 6 months (3) and after 2 years (4). Squares BPD, triangles LAGB, circles LGCP. 22:4n-6: Exam: $F=5.3, p=0.008$, Oper x Exam: $F=4.0, p=0.006$. 22:5n-3: Exam: $F=5.6, p<0.006$, Oper $x$ Exam: $F=2.8, p<0.034$.
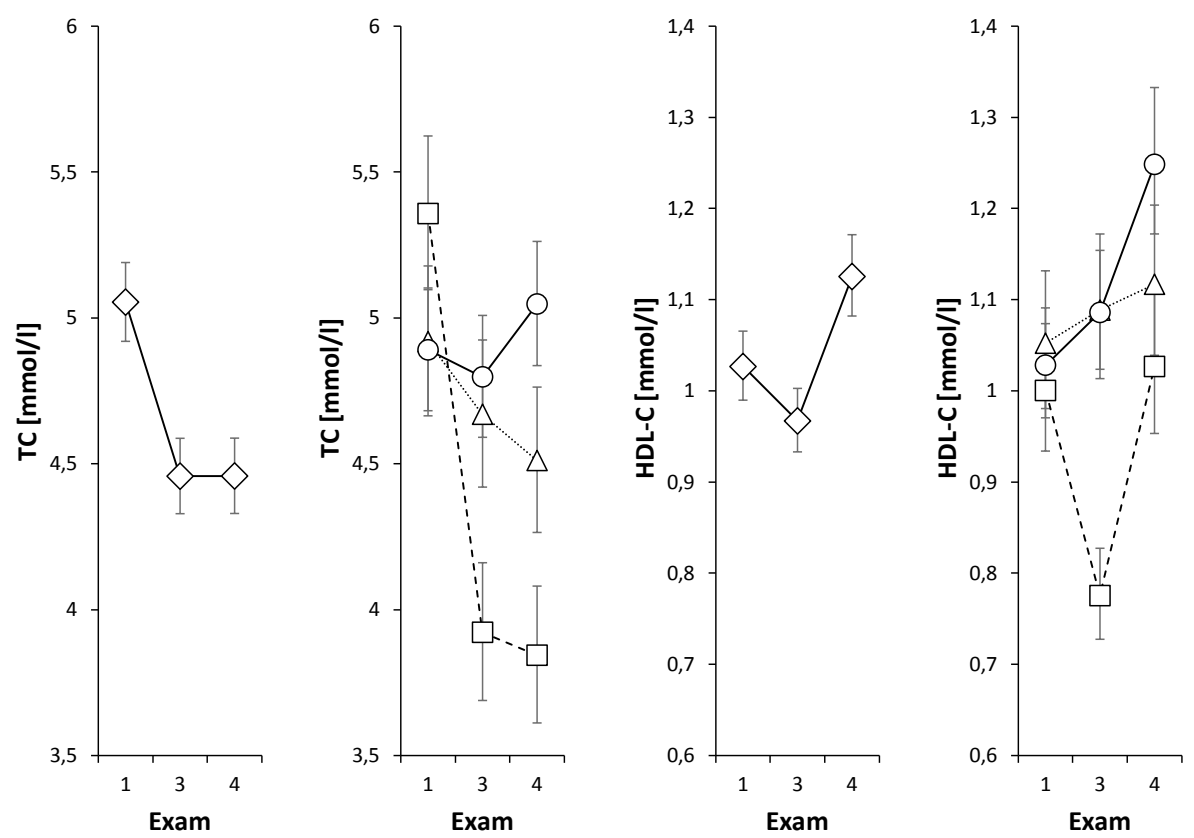

Fig. 4. Total cholesterol and HDL-cholesterol in serum before the surgery (1), after 6 months (3) and after 2 years (4). Squares BPD, triangles LAGB, circles LGCP. TC: Exam: $F=13.8, p<0.001$, Oper $x$ Exam: $F=8.8, p<0.001$. HDL-C: Exam: $F=8.3, p<0.001$, Oper $x$ Exam: $F=4.7, p=0.002$.

For serum lipids, a significant decrease in total cholesterol was found in the BPD group only, while HDL cholesterol increased significantly after LGCP (Table 4, Fig. 4). The inflammatory marker hsCRP decreased significantly in the BPD group, but only an insignificant decrease was found in the LAGB and LGCP groups (Table 4, Fig. 5).
A significantly higher remission of type 2 diabetes was found after biliopancreatic diversion (in 7 of 8 subjects) in comparison with lapascopic gastric banding (6 of 9 subjects) and laparoscopic greater curvature plication (0/12), $\mathrm{P}=0.0004$. Most of the subjects in the gastric plication group were treated by metformin only ( 7 of 12 subjects). 

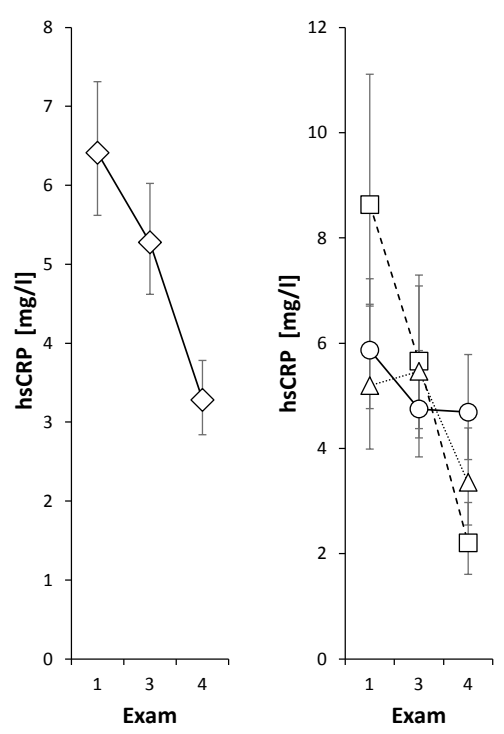

Fig. 5. Hs C-reactive protein in serum before the surgery (1), after 6 months (3) and after 2 years (4). Squares BPD, triangles LAGB, circles LGCP. Exam: $F=12.8, p<0.001$, Oper $x$ Exam: $\mathrm{F}=3.7, \mathrm{p}=0.01$.

Table 1. Characteristic of the subjects and effect of bariatric procedures.

\begin{tabular}{|c|c|c|c|c|c|c|}
\hline \multirow{2}{*}{ Parameter } & \multirow{2}{*}{ Surgery } & \multicolumn{3}{|c|}{ Exam } & \multicolumn{2}{|c|}{ ANOVA ** } \\
\hline & & $1^{*}$ & 3 & 4 & F-ratio & p-value \\
\hline Age & BPD & $52(48.8,54.8)$ & ----- & ----- & & \\
\hline \multirow[t]{2}{*}{ (years) } & LAGB & $61.5(59.4,63.5)$ & ----- & ----- & 9.8 & $<0.001$ \\
\hline & GP & $53.6(51.2,55.8)$ & ----- & ----- & & \\
\hline \multirow{3}{*}{$\begin{array}{l}\text { Height } \\
(\mathrm{cm})\end{array}$} & BPD & $166(163,169)$ & ---- & ----- & & \\
\hline & LAGB & $164(161,167)$ & ----- & ----- & 0.5 & 0.603 \\
\hline & GP & $166(164,168)$ & ---- & ----- & & \\
\hline \multirow{3}{*}{$\begin{array}{l}B M I \\
\left(\mathrm{~kg} / \mathrm{m}^{2}\right)\end{array}$} & BPD & $46.4(44.9,47.9)$ & $38.5(37.5,39.6)$ & $36(35,37)$ & & \\
\hline & LAGB & $44.8(43.5,46.1)$ & $39.6(38.6,40.7)$ & $38.4(37.5,39.4)$ & $5.5^{* *}$ & 0.001 \\
\hline & GP & $38.7(37.8,39.7)$ & $34.7(34.1,35.4)$ & $35.9(35.2,36.7)$ & & \\
\hline \multirow{3}{*}{$\begin{array}{l}\text { Weight } \\
(\mathrm{kg})\end{array}$} & BPD & $127(124,131)$ & $106(103,109)$ & $99.1(96.5,102)$ & & \\
\hline & LAGB & $120(117,123)$ & $107(104,109)$ & $104(101,106)$ & 6.5 & $<0.001$ \\
\hline & GP & $107(104,109)$ & $95.7(93.8,97.6)$ & $99(97.1,101)$ & & \\
\hline \multirow{3}{*}{$\begin{array}{l}\text { Waist } \\
(\mathrm{cm})\end{array}$} & BPD & $124(121,127)$ & $112(109,115)$ & $108(105,111)$ & & \\
\hline & LAGB & $125(122,128)$ & $117(115,120)$ & $114(112,117)$ & 2.6 & 0.049 \\
\hline & GP & $111(109,113)$ & $103(101,105)$ & $106(104,108)$ & & \\
\hline \multirow{3}{*}{$\begin{array}{l}\text { Hip } \\
(\mathrm{cm})\end{array}$} & BPD & $141(138,144)$ & $128(125,131)$ & $122(119,125)$ & & \\
\hline & LAGB & $138(136,141)$ & $133(130,135)$ & $130(127,132)$ & 4.3 & 0.005 \\
\hline & GP & $129(127,131)$ & $120(118,123)$ & $124(122,127)$ & & \\
\hline \multirow{3}{*}{$\begin{array}{l}\text { Body fat } \\
(\%)\end{array}$} & BPD & $53(51.8,54.2)$ & $45.6(44.3,46.9)$ & $43.7(42.2,45.2)$ & & \\
\hline & LAGB & $51.9(50.8,53)$ & $48.9(47.7,50)$ & $48.3(47.2,49.5)$ & 6.9 & $<0.001$ \\
\hline & GP & $50.8(49.8,51.7)$ & $47.7(46.6,48.7)$ & $47.7(46.6,48.7)$ & & \\
\hline \multirow{3}{*}{$\begin{array}{l}S B P \\
(m m H g)\end{array}$} & BPD & $128(121,135)$ & $115(109,122)$ & $117(110,124)$ & & \\
\hline & LAGB & $130(123,138)$ & $120(113,127)$ & $117(110,124)$ & 1.3 & 0.285 \\
\hline & GP & $131(125,137)$ & $118(112,124)$ & $118(112,124)$ & & \\
\hline \multirow{3}{*}{$\begin{array}{l}D B P \\
(m m ~ H g)\end{array}$} & BPD & $80.1(76,84.4)$ & $71.4(67.6,75.3)$ & $68(64.4,71.8)$ & & \\
\hline & LAGB & $81.9(77.8,86.3)$ & $82.6(78.4,87)$ & $75.1(70.9,79.6)$ & 2.9 & 0.065 \\
\hline & GP & $79.5(76.2,83)$ & $73(69.9,76.3)$ & $73(69.9,76.3)$ & & \\
\hline \multirow{3}{*}{$\begin{array}{l}H R \\
(/ \min )\end{array}$} & BPD & $82.3(78.4,86.3)$ & $75(71.4,78.7)$ & $70.8(67.5,74.4)$ & & \\
\hline & LAGB & $78.5(74.7,82.5)$ & $72.9(69.4,76.7)$ & $71.6(68.1,75.3)$ & 0.7 & 0.512 \\
\hline & GP & $75.7(72.5,78.9)$ & $71(68,74.2)$ & $71(68,74.2)$ & & \\
\hline
\end{tabular}

*mean (lower limit of CI, upper limit of CI at $95 \%$ level); ** ANOVA model Oper x Exam; SBP, systolic blood pressure; DBP, diastolic blood pressure; HR, heart rate. 
Table 2. Changes in adipose tissue saturated and monounsaturated fatty acids.

\begin{tabular}{|c|c|c|c|c|c|c|c|c|c|c|}
\hline \multirow{3}{*}{ Variable } & \multirow{3}{*}{ Oper } & \multirow{2}{*}{\multicolumn{3}{|c|}{ Exam }} & \multicolumn{6}{|c|}{ ANOVA model } \\
\hline & & & & & \multicolumn{2}{|c|}{ Oper } & \multicolumn{2}{|c|}{ Exam } & \multicolumn{2}{|c|}{ Oper $\times$ Exam } \\
\hline & & 1 & 3 & 4 & F-ratio & p-value & F-ratio & p-value & F-ratio & p-value \\
\hline \multirow[t]{3}{*}{$12: 0$} & BPD & $0.17(0.15,0.2)$ & $0.15(0.13,0.17)$ & $0.24(0.2,0.27)$ & & & & & & \\
\hline & LAGB & $0.17(0.15,0.2)$ & $0.22(0.19,0.25)$ & $0.2(0.18,0.24)$ & 1.3 & 0.278 & 2.7 & 0.073 & 2.7 & 0.037 \\
\hline & LGCP & $0.17(0.15,0.19)$ & $0.17(0.15,0.19)$ & $0.17(0.15,0.19)$ & & & & & & \\
\hline \multirow[t]{3}{*}{$14: 0$} & $\mathrm{BPD}$ & $2.1(1.99,2.21)$ & $1.95(1.85,2.05)$ & $2.28(2.17,2.41)$ & & & & & & \\
\hline & LAGB & $1.96(1.86,2.06)$ & $2.1(2,2.21)$ & $2.03(1.93,2.14)$ & 0.6 & 0.557 & 2 & 0.142 & 2.5 & 0.054 \\
\hline & LGCP & $2.11(2.02,2.2)$ & $2.07(1.98,2.16)$ & $2.07(1.98,2.16)$ & & & & & & \\
\hline \multirow[t]{3}{*}{$14: 1 n-5$} & BPD & $0.2(0.18,0.22)$ & $0.17(0.15,0.19)$ & $0.29(0.25,0.33)$ & & & & & & \\
\hline & LAGB & $0.21(0.19,0.24)$ & $0.26(0.23,0.29)$ & $0.22(0.2,0.25)$ & 0.7 & 0.52 & 2.5 & 0.094 & 6.7 & $<0.001$ \\
\hline & LGCP & $0.23(0.21,0.25)$ & $0.2(0.19,0.23)$ & $0.2(0.19,0.23)$ & & & & & & \\
\hline \multirow[t]{3}{*}{$16: 0$} & $\mathrm{BPD}$ & $22.8(22.2,23.4)$ & $21.8(21.1,22.5)$ & $21.5(20.8,22.1)$ & & & & & & \\
\hline & LAGB & $21.8(21.1,22.4)$ & $21.7(21.1,22.3)$ & $21.3(20.6,21.9)$ & 12.8 & $<0.001$ & 1.6 & 0.206 & 1.2 & 0.323 \\
\hline & LGCP & $23.1(22.7,23.6)$ & $23.4(22.9,23.8)$ & $23.4(22.9,23.8)$ & & & & & & \\
\hline \multirow[t]{3}{*}{$16: 1 n-9$} & $\mathrm{BPD}$ & $0.82(0.78,0.86)$ & $0.89(0.84,0.93)$ & $0.98(0.94,1)$ & & & & & & \\
\hline & LAGB & $0.77(0.74,0.81)$ & $0.78(0.74,0.82)$ & $0.87(0.83,0.92)$ & 7.9 & $<0.001$ & 12.1 & $<0.001$ & 1.5 & 0.21 \\
\hline & LGCP & $0.79(0.76,0.82)$ & $0.82(0.79,0.85)$ & $0.82(0.79,0.85)$ & & & & & & \\
\hline \multirow[t]{3}{*}{$16: 1 n-7$} & BPD & $4.41(3.0,6.83)$ & $4.29(2.89,6.68)$ & $5.28(3.74,7.87)$ & & & & & & \\
\hline & LAGB & $5.26(3.58,8.3)$ & $6.09(4.27,9.39)$ & $5.4(3.7,8.48)$ & 5.8 & 0.005 & 0.9 & 0.412 & 3 & 0.027 \\
\hline & LGCP & $4.83(3.37,7.32)$ & $4.66(3.22,7.11)$ & $4.66(3.22,7.11)$ & & & & & & \\
\hline \multirow[t]{3}{*}{$18: 0$} & BPD & $16.3(15.3,17.5)$ & $18.3(17,19.6)$ & $20.8(19.5,22.2)$ & & & & & & \\
\hline & LAGB & $17.5(16.4,18.7)$ & $19.8(18.6,21.1)$ & $18.8(17.6,20)$ & 8.4 & $<0.001$ & 4.6 & 0.013 & 3.1 & 0.021 \\
\hline & LGCP & $16.3(15.4,17.2)$ & $16.5(15.6,17.4)$ & $16.5(15.6,17.4)$ & & & & & & \\
\hline \multirow[t]{3}{*}{$18: 1 \mathrm{t}$} & BPD & $0.54(0.51,0.58)$ & $0.46(0.44,0.49)$ & $0.43(0.41,0.46)$ & & & & & & \\
\hline & LAGB & $0.49(0.46,0.51)$ & $0.46(0.43,0.48)$ & $0.44(0.41,0.46)$ & 12.7 & $<0.001$ & 11.8 & $<0.001$ & 1 & 0.408 \\
\hline & LGCP & $0.57(0.54,0.6)$ & $0.56(0.53,0.59)$ & $0.56(0.53,0.59)$ & & & & & & \\
\hline \multirow[t]{3}{*}{$18: 1 n-9$} & $\mathrm{BPD}$ & $46.5(45.8,47.1)$ & $47.6(47,48.3)$ & $47.3(46.7,47.9)$ & & & & & & \\
\hline & LAGB & $46.3(45.7,46.9)$ & $46(45.3,46.6)$ & $47.4(46.8,48.1)$ & 0.9 & 0.414 & 1.8 & 0.174 & 2.2 & 0.079 \\
\hline & LGCP & $46.8(46.3,47.4)$ & $46.9(46.4,47.5)$ & $46.9(46.4,47.5)$ & & & & & & \\
\hline \multirow[t]{3}{*}{$18: \ln -7$} & $\mathrm{BPD}$ & $2.72(2.61,2.84)$ & $2.71(2.6,2.83)$ & $2.97(2.85,3.09)$ & & & & & & \\
\hline & LAGB & $2.8(2.69,2.91)$ & $2.84(2.73,2.96)$ & $2.75(2.64,2.87)$ & 13.1 & $<0.001$ & 0.3 & $<0.754$ & 1.9 & $<0.117$ \\
\hline & LGCP & $2.54(2.44,2.63)$ & $2.55(2.46,2.64)$ & $2.55(2.46,2.64)$ & & & & & & \\
\hline \multirow[t]{3}{*}{$20: 0$} & $\mathrm{BPD}$ & $0.068(0.062,0.074)$ & $0.064(0.058,0.07)$ & $0.057(0.053,0.063)$ & & & & & & \\
\hline & LAGB & $0.072(0.066,0.078)$ & $0.064(0.059,0.07)$ & $0.07(0.064,0.076)$ & 1 & 0.382 & 2.3 & 0.108 & 1 & 0.417 \\
\hline & LGCP & $0.066(0.062,0.071)$ & $0.066(0.061,0.071)$ & $0.066(0.061,0.071)$ & & & & & & \\
\hline \multirow[t]{3}{*}{$20: \ln -9$} & BPD & $0.69(0.65,0.74)$ & $0.71(0.67,0.76)$ & $0.76(0.72,0.8)$ & & & & & & \\
\hline & LAGB & $0.69(0.65,0.73)$ & $0.66(0.62,0.71)$ & $0.73(0.69,0.78)$ & 6.1 & 0.004 & 1.4 & 0.257 & 1.1 & 0.391 \\
\hline & LGCP & $0.65(0.62,0.69)$ & $0.65(0.61,0.68)$ & $0.65(0.61,0.68)$ & & & & & & \\
\hline
\end{tabular}

*mean (lower limit of CI, upper limit of CI at $95 \%$ level).

\section{Discussion}

The main result of the study was the significant difference among bariatric procedures in changes of saturated and monounsaturated fatty acids as well as stearoyl CoA desaturase (SCD1) activity evaluated as the product/precursor ratio. The increases in lauric (12:0), myristoleic $(14: 1 n-5)$ and palmitoleic $(16: 1 n-7)$ acid concurrently with the increased activity of SCD1 in the biliopacreatic diversion group suggests lasting enhanced lipogenic activity 2 years after the surgery, in contrast to the LAGB and LGCP subjects. A strong association between the SCD1 product/precursor ratio and mRNA expression has been found in abdominal subcutaneous adipose tissue (Pinnick et al 2012). 
Table 3. Changes in adipose tissue polyunsaturated fatty acids and sums of saturated, monounsaturated and polyunsaturated fatty acids.

\begin{tabular}{|c|c|c|c|c|c|c|c|c|c|c|}
\hline \multirow{3}{*}{ Variable } & \multirow{3}{*}{ Oper } & \multirow{2}{*}{\multicolumn{3}{|c|}{ Exam }} & \multicolumn{6}{|c|}{ ANOVA model } \\
\hline & & & & & \multicolumn{2}{|c|}{ Oper } & \multicolumn{2}{|c|}{ Exam } & \multicolumn{2}{|c|}{ Oper $\times$ Exam } \\
\hline & & 1 & 3 & 4 & F-ratio & p-value & F-ratio & p-value & F-ratio & p-value \\
\hline \multirow[t]{3}{*}{$18: 2 n-6$} & $\mathrm{BPD}$ & $13(12.4,13.5)$ & $12.8(12.3,13.4)$ & $12.5(12,13)$ & & & & & & \\
\hline & LAGB & $13.3(12.8,13.8)$ & $13.1(12.6,13.6)$ & $12.5(12.1,13)$ & 0.4 & 0.658 & 1.9 & 0.164 & 0.4 & 0.793 \\
\hline & LGCP & $12.9(12.5,13.4)$ & $12.4(12,12.9)$ & $12.4(12,12.9)$ & & & & & & \\
\hline \multirow[t]{3}{*}{$18: 3 n-6$} & BPD & $0.03(0.025,0.036)$ & $0.025(0.021,0.03)$ & $0.036(0.03,0.043)$ & & & & & & \\
\hline & LAGB & $0.041(0.034,0.049)$ & $0.041(0.035,0.049)$ & $0.038(0.032,0.046)$ & 3.1 & 0.053 & 0.5 & 0.632 & 0.9 & 0.445 \\
\hline & LGCP & $0.037(0.032,0.043)$ & $0.036(0.031,0.042)$ & $0.036(0.031,0.042)$ & & & & & & \\
\hline \multirow[t]{3}{*}{$18: 3 n-3$} & BPD & $0.81(0.76,0.85)$ & $0.69(0.65,0.73)$ & $0.71(0.67,0.75)$ & & & & & & \\
\hline & LAGB & $0.8(0.76,0.84)$ & $0.8(0.76,0.84)$ & $0.76(0.72,0.8)$ & 1.7 & 0.185 & 4.7 & 0.013 & 1.7 & 0.16 \\
\hline & LGCP & $0.78(0.74,0.82)$ & $0.72(0.69,0.75)$ & $0.72(0.69,0.75)$ & & & & & & \\
\hline \multirow[t]{3}{*}{$20: 2 n-6$} & BPD & $0.25(0.24,0.27)$ & $0.26(0.25,0.28)$ & $0.27(0.26,0.29)$ & & & & & & \\
\hline & LAGB & $0.26(0.25,0.27)$ & $0.25(0.24,0.27)$ & $0.24(0.23,0.26)$ & 12.6 & $<0.001$ & 0 & 0.961 & 1.1 & 0.371 \\
\hline & LGCP & $0.23(0.22,0.24)$ & $0.23(0.22,0.24)$ & $0.23(0.22,0.24)$ & & & & & & \\
\hline \multirow[t]{3}{*}{$20: 3 n-6$} & BPD & $0.38(0.36,0.41)$ & $0.38(0.35,0.41)$ & $0.46(0.42,0.5)$ & & & & & & \\
\hline & LAGB & $0.35(0.33,0.38)$ & $0.32(0.3,0.34)$ & $0.36(0.33,0.38)$ & 9.6 & $<0.001$ & 3.3 & 0.044 & 2 & 0.111 \\
\hline & LGCP & $0.35(0.33,0.37)$ & $0.35(0.33,0.37)$ & $0.35(0.33,0.37)$ & & & & & & \\
\hline \multirow[t]{3}{*}{$20: 4 n-6$} & BPD & $0.57(0.55,0.6)$ & $0.56(0.53,0.59)$ & $0.61(0.58,0.64)$ & & & & & & \\
\hline & LAGB & $0.59(0.56,0.62)$ & $0.58(0.55,0.61)$ & $0.6(0.57,0.62)$ & 2.1 & 0.128 & 1.5 & 0.235 & 0.5 & 0.731 \\
\hline & LGCP & $0.57(0.54,0.59)$ & $0.55(0.53,0.57)$ & $0.55(0.53,0.57)$ & & & & & & \\
\hline \multirow[t]{3}{*}{$20: 5 n-3$} & BPD & $0.054(0.048,0.06)$ & $0.045(0.04,0.051)$ & $0.053(0.047,0.06)$ & & & & & & \\
\hline & LAGB & $0.057(0.05,0.064)$ & $0.051(0.046,0.057)$ & $0.051(0.045,0.057)$ & 14.7 & $<0.001$ & 3.2 & 0.047 & 0.6 & 0.674 \\
\hline & LGCP & $0.071(0.065,0.077)$ & $0.062(0.057,0.068)$ & $0.062(0.057,0.068)$ & & & & & & \\
\hline \multirow[t]{3}{*}{$22: 4 n-6$} & $\mathrm{BPD}$ & $0.24(0.23,0.26)$ & $0.27(0.25,0.29)$ & $0.33(0.3,0.36)$ & & & & & & \\
\hline & LAGB & $0.22(0.21,0.24)$ & $0.2(0.19,0.21)$ & $0.23(0.21,0.24)$ & 15.3 & $<0.001$ & 5.3 & 0.008 & 4 & 0.006 \\
\hline & LGCP & $0.23(0.22,0.24)$ & $0.24(0.23,0.25)$ & $0.24(0.23,0.25)$ & & & & & & \\
\hline \multirow[t]{3}{*}{$22: 5 n-6$} & BPD & $0.037(0.033,0.042)$ & $0.037(0.033,0.043)$ & $0.043(0.037,0.05)$ & & & & & & \\
\hline & LAGB & $0.034(0.03,0.038)$ & $0.031(0.028,0.035)$ & $0.033(0.03,0.038)$ & 7.1 & 0.002 & 0.8 & 0.45 & 0.5 & 0.762 \\
\hline & LGCP & $0.029(0.026,0.031)$ & $0.031(0.028,0.034)$ & $0.031(0.028,0.034)$ & & & & & & \\
\hline \multirow[t]{3}{*}{$22: 5 n-3$} & BPD & $0.24(0.22,0.25)$ & $0.23(0.21,0.24)$ & $0.29(0.27,0.31)$ & & & & & & \\
\hline & LAGB & $0.23(0.22,0.25)$ & $0.21(0.19,0.22)$ & $0.24(0.22,0.25)$ & 5.8 & 0.005 & 5.6 & 0.006 & 2.8 & 0.034 \\
\hline & LGCP & $0.26(0.24,0.27)$ & $0.27(0.26,0.29)$ & $0.27(0.26,0.29)$ & & & & & & \\
\hline \multirow[t]{3}{*}{$22: 6 n-3$} & BPD & $0.14(0.13,0.15)$ & $0.12(0.11,0.13)$ & $0.14(0.12,0.15)$ & & & & & & \\
\hline & LAGB & $0.13(0.12,0.14)$ & $0.12(0.11,0.13)$ & $0.12(0.11,0.13)$ & 14.2 & $<0.001$ & 0.4 & 0.669 & 0.9 & 0.467 \\
\hline & LGCP & $0.15(0.14,0.17)$ & $0.16(0.15,0.18)$ & $0.16(0.15,0.18)$ & & & & & & \\
\hline \multirow[t]{3}{*}{ SFA } & BPD & $27.5(26.7,28.3)$ & $26(25,26.9)$ & $25.7(24.8,26.6)$ & & & & & & \\
\hline & LAGB & $26.9(26.1,27.7)$ & $26.6(25.8,27.4)$ & $26.3(25.5,27.1)$ & 24.1 & $<0.001$ & 1.5 & 0.236 & 1.1 & 0.359 \\
\hline & LGCP & $29(28.4,29.6)$ & $29.2(28.6,29.8)$ & $29.2(28.6,29.8)$ & & & & & & \\
\hline \multirow[t]{3}{*}{ MFA } & $\mathrm{BPD}$ & $56.3(55.5,57.1)$ & $57.3(56.5,58.2)$ & $58.3(57.5,59.2)$ & & & & & & \\
\hline & LAGB & $56.6(55.8,57.4)$ & $57.1(56.3,57.9)$ & $58(57.2,58.9)$ & 9.4 & $<0.001$ & 4 & 0.024 & 1 & 0.431 \\
\hline & LGCP & $55.5(54.9,56.2)$ & $55.5(54.8,56.2)$ & $55.5(54.8,56.2)$ & & & & & & \\
\hline PUFAn-6 & $\mathrm{BPD}$ & $14.6(13.9,15.2)$ & $14.5(13.8,15.2)$ & $14.3(13.7,15)$ & & & & & & \\
\hline & LAGB & $14.5(13.9,15.1)$ & $14.2(13.6,14.8)$ & $13.7(13.2,14.3)$ & 0.5 & 0.586 & 0.4 & 0.664 & 0.5 & 0.771 \\
\hline & LGCP & $14.6(14,15.1)$ & $14.4(13.9,15)$ & $14.4(13.9,15)$ & & & & & & \\
\hline PUFAn-3 & BPD & $1.24(1.19,1.29)$ & $1.09(1.05,1.13)$ & $1.19(1.15,1.24)$ & & & & & & \\
\hline & LAGB & $1.24(1.19,1.29)$ & $1.17(1.13,1.21)$ & $1.16(1.12,1.21)$ & 3.7 & 0.032 & 7.2 & 0.002 & 1.5 & 0.204 \\
\hline & LGCP & $1.27(1.22,1.31)$ & $1.22(1.19,1.27)$ & $1.22(1.19,1.27)$ & & & & & & \\
\hline
\end{tabular}

*mean (lower limit of $\mathrm{CI}$, upper limit of $\mathrm{CI}$ at $95 \%$ level). 
Table 4. Changes in blood lipids and hsCRP.

\begin{tabular}{|c|c|c|c|c|c|c|c|c|c|c|}
\hline \multirow{3}{*}{ Variable } & \multirow{3}{*}{ Oper } & \multirow{2}{*}{\multicolumn{3}{|c|}{ Exam }} & \multicolumn{6}{|c|}{ ANOVA model } \\
\hline & & & & & \multicolumn{2}{|c|}{ Oper } & \multicolumn{2}{|c|}{ Exam } & \multicolumn{2}{|c|}{ Oper $\times$ Exam } \\
\hline & & 1 & 3 & 4 & F-ratio & p-value & F-ratio & p-value & F-ratio & p-value \\
\hline \multirow[t]{3}{*}{$\mathrm{TC}$} & BPD & $5.36(5.1,5.62)$ & $3.92(3.69,4.16)$ & $3.84(3.61,4.08)$ & & & & & & \\
\hline & LAGB & $4.92(4.66,5.18)$ & $4.67(4.42,4.92)$ & $4.51(4.26,4.76)$ & 8.7 & $<0.001$ & 13.8 & $<0.001$ & 8.8 & $<0.001$ \\
\hline & LGCP & $4.89(4.68,5.1)$ & $4.8(4.59,5.01)$ & $4.8(4.59,5.01)$ & & & & & & \\
\hline \multirow[t]{3}{*}{ HDL } & BPD & $1(0.934,1.07)$ & $0.775(0.728,0.827)$ & $1.03(0.953,1.11)$ & & & & & & \\
\hline & LAGB & $1.05(0.981,1.13)$ & $1.09(1.01,1.17)$ & $1.12(1.04,1.2)$ & 12.7 & $<0.001$ & 8.3 & $<0.001$ & 4.7 & 0.002 \\
\hline & LGCP & $1.03(0.97,1.09)$ & $1.09(1.02,1.15)$ & $1.09(1.02,1.15)$ & & & & & & \\
\hline \multirow[t]{3}{*}{ TG } & BPD & $1.24(1.07,1.44)$ & $1.42(1.22,1.66)$ & $1.01(0.876,1.17)$ & & & & & & \\
\hline & LAGB & $1.68(1.44,1.99)$ & $1.31(1.11,1.54)$ & $1.4(1.2,1.64)$ & 2.2 & 0.123 & 3.5 & 0.038 & 1.8 & 0.143 \\
\hline & LGCP & $1.38(1.22,1.57)$ & $1.08(0.957,1.21)$ & $1.08(0.957,1.21)$ & & & & & & \\
\hline \multirow[t]{3}{*}{ hsCRP } & BPD & $8.63(6.7,11.1)$ & $5.66(4.38,7.29)$ & $2.21(1.61,2.97)$ & & & & & & \\
\hline & LAGB & $5.19(3.99,6.74)$ & $5.47(4.2,7.09)$ & $3.36(2.54,4.39)$ & 0.2 & 0.819 & 12.8 & $<0.001$ & 3.7 & 0.01 \\
\hline & LGCP & $5.87(4.76,7.22)$ & $4.75(3.84,5.86)$ & $4.75(3.84,5.86)$ & & & & & & \\
\hline
\end{tabular}

*mean (lower limit of CI, upper limit of CI at $95 \%$ level); TC, total cholesterol; HDL, HDL cholesterol; TG, triglycerides; hsCRP, high sensitivity C-reactive protein.

The relationship of circulating palmitoleate to insulin sensitivity has been broadly discussed in animals as well as in humans, but with unequivocal conclusions. A large study in Finnish men using proton magnetic spectroscopy for analysis of serum FA profiles showed that an increased percentage of saturated but also n-7 and n-9 monounsaturated FA in serum were biomarkers for an increased risk of the development of hyperglycemia and type 2 diabetes (Mahendran et al. 2013). On the other hand, Stefan et al. (2010) showed a positive correlation of serum palmitoleate (16:1n-7) with insulin sensitivity measured by oGTT and euglycemic hyperinsulinemic clamp in subjects with increased risk for type 2 diabetes. Additionally, no influence of palmitoleate availability on insulin sensitivity assessed by euglycemic hyperinsulinemic clamp was reported in obese insulin sensitive and insulin resistant subjects (Fabbrini et al. 2011).

We found the highest lipogenesis in subjects with the highest weight loss after 2 years, i.e. in the BPD group. This corresponds to the results of the panEuropean Diogenes study, where obese subjects were treated by a low calorie diet for two months followed by a six-month weight maintenance regimen (Larsen et al. 2010). Significant higher weight loss after 8 months was found in subjects with lower baseline monounsaturated fatty acids (14:1n-5, 16:1n-7 and trans 16:1n-7) in adipose tissue triglycerides (Kunešová et al. 2012). When evaluating the relationship of fatty acid composition with mRNA expression from the same biopsy of adipose tissue in the Diogenes study, the central role of myristoleic acid after LCD was associated with the down-regulation of genes involved in fatty acid biosynthesis (SCD, FADS1 and FADS2). After the weight maintenance period, there was a significant positive relationship of $14: 1 \mathrm{n}-5$ with genes involved in de novo lipogenesis (AACS, FASN, SCD, FADS1, FADS2 and ELOVL5) only in subjects with continuing weight loss in contrast to subjects who regained weight (Monastier et al. 2015), suggesting enhanced lipogenesis in subjects with continuing weight loss.

The role of palmitoleate as a possible lipokine has been widely discussed in the past few years. The recent review of Hodson and Karpe (2013) summarized the current knowledge on palmitoleate and emphasized the different roles of palmitoleate in blood and adipose tissue. The localization of adipose tissue was shown to play a major role in the release of palmitoleic acid, with a higher contribution from gluteofemoral subcutaneous fat in comparison with abdominal subcutaneous fat. This difference may be the result of enhanced SCD1 mRNA expression in gluteal adipose tissue (Pinnick et al. 2012). In this context, the increase of myristoleic and palmitoleic acids in abdominal subcutaneous fat found in our study could reflect a positive metabolic effect of BPD. A similar result was found in a study comparing overweight and obese subjects in which lower monounsaturated FAs and palmitoleic acid content were found in subcutaneous and visceral adipose tissue, respectively, with increasing adiposity (Garaulet et al. 2011). 
Polyunsaturated fatty acids are a minor FA component of triglycerides in adipose tissue. An enhanced percentage of docosatetraenoic acid (22:4n-6) in AT was shown in the BPD group after 2 years, in contrast to the LAGB and LGCP groups. This could be the result of a better supply of this FA in food. Better resorption is less probable due to the malabsorptive character of the surgery. Enhanced elongation of arachidonic acid (20:4n-6) or reduced oxidation of $n-6$ PUFA could be other contributing factors.

The decreased percentage of docosapentaenoic acid (22:5n-3) in the BPD and LAGB groups 6 months after surgery could reflect the increased oxidation of $n-3$ PUFA or their lower supply from circulation due to decreased intake or absorption. After 2 years, the levels returned to baseline.

\section{Type 2 DM remission}

We found significant differences in type 2 diabetes mellitus remission (T2DMR) after individual bariatric procedures. BPD resulted in the highest remission followed by LAGB, while in the LGCP group most subjects were treated by metformin two years after the surgery. Robert et al. (2013) did not find differences in T2DMR when comparing Roux-en-Y gastric bypass, sleeve gastrectomy and LAGB. They showed rather that preoperative BMI $\leq 50 \mathrm{~kg} / \mathrm{m}^{2}$, duration of type 2 diabetes $\leq 4$ years, glycated hemoglobin $\leq 7.1 \%$, fasting glucose $<1.14 \mathrm{~g} / 1$ and absence of insulin therapy were positive predictors of diabetes remission. Concurrently, a short duration of diabetes and good preoperative glycemic control increased the rates of T2DM remission. Their results suggest that preoperative metabolic data could be of greater importance than the choice of bariatric procedure. Steven et al. (2015) did not distinguish between individual bariatric procedures, but patients in that study mostly (63\%) underwent Roux-en-Y gastric bypass. Significant predictors were maximum percentage of weight loss and baseline HbAlc. Preoperative BMI, diabetes duration and age were not significant predictors. This result was also supported by a meta-analysis that found that diabetes remission is unrelated to basal BMI (Panunzi et al. 2015).

\section{Conclusion}

Significant differences in the fatty acid composition of adipose tissue triglycerides were found among three methods of bariatric surgery. Biliopancreatic diversion lead to an increase of saturated (12:0, 18:0) and monounsaturated (14:1n-5 and 16:1n-7) fatty acids and stearoyl CoA desaturase 1 after 2 years, suggesting enhanced lipogenesis. The highest type 2 diabetes mellitus remission was found after BPD. In contrast, minor changes were found after laparoscopic gastric banding and laparoscopic greater curvature plication.

\section{Conflict of Interest}

There is no conflict of interest.

\section{Acknowledgements}

The study was supported by grant IGA NT-13735-4, Ministry of Health of the Czech Republic.

\section{References}

BRADNOVA O, KYROU I, HAINER V, VCELAK J, HALKOVA T, SRAMKOVA P, DOLEZALOVA K, FRIED M, MCTERNAN P, KUMAR S, HILL M, KUNESOVA M, BENDLOVA B, VRBIKOVA J: Laparoscopic greater curvature plication in morbidly obese women with type 2 diabetes: effects on glucose homeostasis, postprandial triglyceridemia and selected gut hormones. Obes Surg 24: 718-726, 2014.

BUCHWALD H, AVIDOR Y, BRAUNWALD E, JENSEN MD, PORIES W, FAHRBACH K, SCHOELLES K: Bariatric surgery: a systematic review and meta-analysis. JAMA 292: 1724-1737, 2004.

BUCHWALD H, ESTOK R, FAHRBACH K, BANEL D, JENSEN MD, PORIES WJ, BANTLE JP, SLEDGE I: Weight and type 2 diabetes after bariatric surgery: systematic review and meta-analysis. Am J Med 122: 248-256, 2009.

BUSE JB, CAPRIO S, CEFALU WT, CERIELLO A, DEL PRATO S, INZUCCHI SE, MCLAUGHLIN S, PHILLIPS GL 2ND, ROBERTSON RP, RUBINO F, KAHN R, KIRKMAN MS: How do we define cure of diabetes? Diabetes Care 32: 2133-2135, 2009.

CALVANI R, MICCHELI A, CAPUANI G, TOMASSINI MICCHELI A, PUCCETTI C, DELFINI M, IACONELLI A, NANNI G, MINGRONE G: Gut microbiome-derived metabolites characterize a peculiar obese urinary metabotype. Int J Obes (Lond) 34: 1095, 2010. 
CARLSON LA: Extraction of lipids from human whole serum and lipoproteins and from rat liver tissue with methylene chloride-methanol: a comparison with extraction with chloroform-methanol. Clin Chim Acta 149: 89-93, 1985.

CLEMENTE-POSTIGO M, ROCA-RODRIGUEZ MDEL M, CAMARGO A, OCAÑA-WILHELMI L, CARDONA F, TINAHONES FJ: Lipopolysaccharide and lipopolysaccharide-binding protein levels and their relationship to early metabolic improvement after bariatric surgery. Surg Obes Relat Dis 11: 933-939, 2015.

FABBRINI E, MAGKOS F, SU X, ABUMRAD NA, NEJEDLY N, COUGHLIN CC, OKUNADE AL, PATTERSON BW, KLEIN S: Insulin sensitivity is not associated with palmitoleate availability in obese humans. $J$ Lipid Res 52: 808-812, 2011.

FERRANNINI E, CAMASTRA S, ASTIARRAGA B, NANNIPIERI M, CASTRO-PEREZ J, XIE D, WANG L, CHAKRAVARTHY M, HAEUSLER RA: Increased bile acid synthesis and deconjugation after biliopancreatic diversion. Diabetes in press 2015.

FOLCH J, LEES M, SLOANE STANLEY GH: A simple method for the isolation andpurification of total lipides from animal tissues. $J$ Biol Chem 226: 497-509, 1957.

FRIED M, DOLEZALOVA K, BUCHWALD JN, MCGLENNON TW, SRAMKOVA P, RIBARIC G: Laparoscopic greater curvature plication (LGCP) for treatment of morbid obesity in a series of 244 patients. Obes Surg 22: 1298-1307, 2012.

GARAULET M, HERNANDEZ-MORANTE JJ, TEBAR FJ, ZAMORA S: Relation between degree of obesity and site-specific adipose tissue fatty acid composition in a Mediterranean population. Nutrition 27: 170-176, 2011.

HILL M, PAŘÍZEK A, CIBULA D, KANCHEVA R, JIRÁSEK JE, JIRKOVSKÁ M, VELÍKOVÁ M, KUBÁTOVÁ J, KLÍMKOVÁ M, PAŠKOVÁ A, ZIŽKA Z, KANCHEVA L, KAZIHNITKOVÁ H, ZAMRAZILOVÁ L, STÁRKA L: Steroid metabolome in plasma from the umbilical artery, umbilical vein, maternal cubital vein and in amniotic fluid in normal and preterm labor. J Steroid Biochem Mol Biol 121: 594-610, 2010.

HODSON L, KARPE F: Is there something special about palmitoleate? Curr OpinClin Nutr Metab Care 16: 225-231, 2013.

KUNEŠOVÁ M, HLAVATÝ P, TVRZICKÁ E, STAŇKOVÁ B, KALOUSKOVÁ P, VIGUERIE N, LARSEN TM, VAN BAAK MA, JEBB SA, MARTINEZ JA, PFEIFFER AF, KAFATOS A, HANDJIEVA-DARLENSKA T, HILL M, LANGIN D, ZÁK A, ASTRUP A, SARIS WH: Fatty acid composition of adipose tissue triglycerides after weight loss and weight maintenance: the DIOGENES study. Physiol Res 61: 597-607, 2012.

LARSEN TM, DALSKOV SM, VAN BAAK M, JEBB SA, PAPADAKI A, PFEIFFER AF, MARTINEZ JA, HANDJIEVA-DARLENSKA T, KUNESOVA M, PIHLSGARD M, STENDERS, HOLST C, SARIS WH, ASTRUP A: Diets with high or low protein content and glycaemic index for weight-loss maintenance. $N$ Engl J Med 363: 2102-2113, 2010.

MAHENDRAN Y, CEDERBERG H, VANGIPURAPU J, KANGAS AJ, SOININEN P, KUUSISTO J, UUSITUPA M, ALA-KORPELA M, LAAKSO M: Glycerol and fatty acids in serum predict the development of hyperglycemia and type 2 diabetes in Finnish men. Diabetes Care 36: 3732-3738, 2013.

MELOUN M, HILL M, MILITKY J, KUPKA K: Transformation in the PC-aided biochemical data analysis. Clin Chem Lab Med 38: 553-559, 2000.

MELOUN M, MILITKY J, HILL M, BRERETON RG: Crucial problems in regression modelling and their solutions. Analyst 127: 433-450, 2002.

MELOUN M, HILL M, MILITKY J, VRBIKOVA J, STANICKA S, SKRHA J: New methodology of influential point detection in regression model building for the prediction of metabolic clearance rate of glucose. Clin Chem Lab Med 42: 311-322, 2004.

MONTASTIER E, VILLA-VIALANEIX N, CASPAR-BAUGUIL S, HLAVATY P, TVRZICKA E, GONZALEZ I, SARIS WH, LANGIN D, KUNESOVA M, VIGUERIE N: System model network for adipose tissue signatures related to weight changes in response to calorie restriction and subsequent weight maintenance. PLoS Comput Biol 11: e1004047, 2015.

OKADA T, FURUHASHI N, KUROMORI Y, MIYASHITA M, IWATA F, HARADA K: Plasma palmitoleic acid content and obesity in children. Am J Clin Nutr 82: 747-750, 2005. 
PANUNZI S, DE GAETANO A, CARNICELli A, MINGRONE G: Predictors of remission of diabetes mellitus in severely obese individuals undergoing bariatric surgery: do BMI or procedure choice matter? A meta-analysis. Ann Surg 261: 459-467, 2015.

PINNICK KE, NEVILLE MJ, FIELDING BA, FRAYN KN, KARPE F, HODSON L: Gluteofemoral adipose tissue plays a major role in production of the lipokine palmitoleate in humans. Diabetes 61: 1399-1403, 2012.

PLOURDE CÉ, GRENIER-LAROUCHE T, CARON-DORVAL D, BIRON S, MARCEAU S, LEBEL S, BIERTHO L, TCHERNOF A, RICHARD D, CARPENTIER AC: Biliopancreatic diversion with duodenal switch improves insulin sensitivity and secretion through caloric restriction. Obesity (Silver Spring) 22: 1838-1846, 2014.

ROBERT M, FERRAND-GAILLARD C, DISSE E, ESPALIEU P, SIMON C, LAVILLE M, GOUILLAT C, THIVOLET C: Predictive factors of type 2 diabetes remission 1 year after bariatric surgery: impact of surgical techniques. Obes Surg 23: 770-775, 2013.

SCHEEN AJ, DE FLINES J, DE ROOVER A, PAQUOT N: Bariatric surgery in patiens with type 2 diabetes: benefits, risks, indications and perspectives. Diabetes Metab 35: 537-543, 2009.

STEFAN N, KANTARTZIS K, CELEBI N, STAIGER H, MACHANN J, SCHICK F, CEGAN A, ELCNEROVA M, SCHLEICHER E, FRITSCHE A, HÄRING HU: Circulating palmitoleate strongly and independently predicts insulin sensitivity in humans. Diabetes Care 33: 405-407, 2010.

STEVEN S, CAREY PE, SMALL PK, TAYLOR R: Reversal of Type 2 diabetes after bariatric surgery is determined by the degree of achieved weight loss in both short- and long-duration diabetes. Diabet Med 32: 47-53, 2015.

SVANE MS, MADSBAD S: Bariatric surgery - effects on obesity and related co-morbidities. Curr Diabetes Rev 10: 208-214, 2014.

TALEBPOUR M, MOTAMEDI SM, TALEBPOUR A, VAHIDI H: Twelve year experience of laparoscopic gastric plication in morbid obesity: development of the technique and patient outcomes. Ann Surg Innov Res 6: 7, 2012.

TSOLI M, CHRONAIOU A, KEHAGIAS I, KALFARENTZOS F, ALEXANDRIDES TK: Hormone changes and diabetes resolution after biliopancreatic diversion and laparoscopic sleeve gastrectomy: a comparative prospective study. Surg Obes Relat Dis 9: 667-677, 2013. 